\title{
Simple procedures of choice in multicriteria problems without precise information about the alternatives' values
}

\author{
Paula Sarabando ${ }^{\mathrm{a}, *}$, Luís C. Dias ${ }^{\mathrm{b}}$ \\ a INESC Coimbra and Departamento de Matemática, Instituto Superior Politécnico de Viseu, Campus Politécnico de Viseu, 3504-510 Viseu, Portugal \\ ${ }^{\mathrm{b}}$ INESC Coimbra and Faculdade de Economia da Universidade de Coimbra, Av Dias da Silva 165, 3004-512 Coimbra, Portugal
}

\section{A R T I C L E I N F O}

Available online 1 April 2010

Keywords:

Multi-criteria decision analysis MAUT/MAVT

Imprecise/incomplete/partial information Ordinal information

Simulation

\begin{abstract}
A B S T R A C T
The additive model of multiattribute value theory is widely used in multicriteria choice problems. But often it is not easy to obtain precise values for the scaling weights or the alternatives' value in each function. Several decision rules which require weaker information, such as ordinal information, have been proposed to select an alternative under these circumstances. We propose new decision rules and test them using Monte-Carlo simulation, considering that there is ordinal information both on the scaling weights and on the alternatives' values. Results show the new rules constitute a good approximation. We provide guidelines about how to use these rules in a context of selecting a subset of the most promising alternatives, considering the contradictory objectives of keeping a low number of alternatives yet not excluding the best one.
\end{abstract}

(c) 2010 Elsevier Ltd. All rights reserved.

\section{Introduction}

There are many approaches to ranking a set of decision alternatives or to selecting the best(s) one(s) taking into account multiple criteria (see, for example, $[5,16]$ for recent comprehensive reviews of multicriteria evaluation approaches). In this paper we address multiattribute utility theory (MAUT)/multiattribute value theory (MAVT) [23], a popular approach that yields a global value assessment for each alternative. Under this technique, a value function for each criterion, must first be built which expresses on a cardinal scale the value associated with each level of the scale used to measure performance in that criterion. In the case of the utility function it is also possible to model different attitudes to risk. The value function may increase or decrease as the level increases (e.g. decreasing in the case of a cost). The most popular model for aggregating multiple value functions is the additive model: under some assumptions [23], the overall value of an alternative is the sum of value functions (one for each evaluation criterion), each weighted by a scale coefficient. We will refer to these scaling coefficients simply as "weights", although noting that they do not directly reflect the intuitive notion of importance of each criterion, as they are contingent on the range for which the value function was defined. This is one of the best known methods for practitioners and researchers; it is

\footnotetext{
* Corresponding author.

E-mail addresses: psarabando@mat.estv.ipv.pt (P. Sarabando), Imcdias@fe.uc.pt (L.C. Dias).
}

simple to understand and its theoretical properties have been thoroughly studied (see for example $[23,44,45]$ ).

It is usually assumed that the exact values for the parameters of multiattribute evaluation models are known or can be elicited from a decision maker. But in many cases this assumption is unrealistic, or there may even be advantages in working with less precise information. There are several reasons why a decision maker might prefer to provide incomplete information $[12,13,25,46]$ : the decision might need to be taken under pressure of time and lack of data; the decision maker might not feel confident in providing precise figures for intangible or non-monetary parameters (e.g., parameters reflecting environmental impacts); the decision maker might have limited scope to process information; the decision maker might not want to reveal his preferences in public or might not want to state his preferences (as they could change during the process); the evaluation of the alternatives in some criteria might result from inaccurate statistics or measurements; the decision maker might consider it difficult to translate qualitative judgments into precise numerical values; the performance of some alternatives might depend on variables whose value is not known at the time of analysis; the information that would set the value of some parameters might be incomplete, not credible, contradictory or controversial. Some of these factors could be mitigated at the expense of time, discussions or money, but the decision maker may want to avoid incurring in these costs. Working with models which require less effort from the decision makers is a way of fostering the adoption of formal methods to assist with decisions. In particular the decision maker might indicate only qualitative or ordinal information, instead of providing exact values for all parameters. 
The concern with working with incomplete information arises naturally in the context of the use of multiattribute value (or utility) functions. Most of the proposed methods deal with imprecision in terms of weights, considering the value of each alternative in each criterion is precisely known (e.g. $[2,13,20,40,42,46])$. There are also methods that address imprecision in performance values (e.g. [21]), or are able to deal with imprecision in weights and in performance values simultaneously (e.g. $[32,35,39,47])$.

The work presented in this paper is motivated by the difficulty of eliciting a precise value for each alternative in each criterion. It addresses imprecision in performance values, both with and without simultaneous imprecision in weights. Eliciting incomplete information about weights and about the value of each alternative in each criterion, although not precise, might be enough to increase the knowledge of the decision maker about the issue under analysis and so lead to the identification of the most promising alternatives.

A research question that arises in this context is to determine the quality of the proposed rules to select an alternative in the context of absence of precise information, compared with an ideal situation in which the values of all parameters of the model are known. Usually this is studied using Monte-Carlo simulations: randomly generating a large number of problems (criteria weights and value of each alternative in each criterion), determining the alternative with the highest multiattribute value, and comparing this alternative with the alternative chosen by the rule being studied, which uses only part of the information. Examples of such comparisons can be found in [4,40-42]. However, these references consider that only the weights are unknown, and it is important to extend this idea to situations where the values of the alternatives in each criterion are also unknown.

This paper presents new rules and simulation studies comparing different choice rules when information about the weight of the criteria and the value of each alternative in each criterion is imprecise. We consider that the available information has an ordinal nature, which Larichev et al. [28] considered can be elicited with greater confidence. For example, the decision maker can indicate that one alternative has higher value than another alternative in one criterion, without quantifying the amount. The decision rules we compare are based on the concept of Rank Order Centroid $([4,43])$, which has been found to perform very well when there is ordinal information on the weights and known cardinal information for the alternatives' values $[4,42]$. The main purpose of our comparisons is then to assess how much the quality of the results degrades when we consider ordinal information on the alternatives' values.

Unlike most previous research, we will not focus solely on the best alternative when comparing rules. Rather than using a rule to identify a single alternative, our aim is to test how the rules behave in a strategy whereby the number of alternatives is progressively reduced [13]. Our aim is to test rules as screening procedures that identify a subset of promising alternatives in an effort to reconcile the contradictory objectives of maintaining a minimum number of alternatives while ensuring that the chosen subset contains the best alternative. These experiments are designed to be comparable with previous studies and so we tested problems of similar dimensions.

In the next section we present some of the approaches reported in the literature that deal with the use of ordinal information and other types of incomplete information. The rules tested are presented in detail in Section 3, which also introduces the mathematical notation. The simulations performed are described in Section 4 and the results are presented in Section 5. Section 6 discusses some conclusions and some ideas for future research.

\section{A review}

There are many methods that work with ordinal information (see, for example, [7]). The decision maker may indicate that one criterion is more important than some of the others, or that one alternative performs better than another in a certain criterion, but not indicate by how much. This concern arises both in MAVT methods and in methods based on different principles. For instance, in the context of outranking methods Bisdorff [6] extended the principle of concordance to the context of ordinal information about criteria weights. The methods QUALIFLEX [34], and ORESTE [37] also enable rankings in several criteria and a ranking of the relative importance of these criteria to be considered.

Other ordinal information methods not based on the idea of a multiattribute value function are verbal decision analysis (VDA) [33], the TOMASO method [31], and distance-based approaches, to cite rather diverse examples. VDA methods (ZAPROS [26] for ranking problems, ORCLASS [27] for classification problems) are designed for problems with a large number of alternatives and a small number of criteria, making very few assumptions about the way the decision maker aggregates preferences. The TOMASO method can also be used for sorting or ranking alternatives evaluated on ordinal scales based on Choquet integrals. Distance-based approaches attempt to find a ranking that is as close as possible (according to a certain distance) to a set of rankings provided as an input. As examples we can cite $[10,11,18]$.

The indirect elicitation of preferences is used in the paradigm of ordinal regression. According to this paradigm, information on holistic preferences concerning a set of reference alternatives is initially obtained and then the parameters for the model that maximize compatibility with this information are inferred. The inferred parameter values are then used to rank the alternatives. In the context of MAVT this class of methods includes UTA [22], MACBETH [3], and GRIP [17]. Rather than inferring precise parameter values, VIP Analysis [13] can use ordinal information to infer constraints on MAVT weights, and then finds the set of conclusions that is compatible with these constraints (robust conclusions). The SMAA method [24] takes the opposite perspective by finding parameter values compatible with potential results, making it possible to find out what parameter values make an alternative the best one. SMAA-O [25] is a variant of SMAA for problems in which criteria are measured on ordinal scales.

To reconstitute the judgement of a decision maker about some alternatives provided as examples it is not necessary to infer numerical constraints or values. Greco et al. [19] presented the Decision Rule Approach, in which preferences are shaped in terms of "if ..., then ..." rules, based on the Dominance-based Rough Set Approach. Also based on the concept of dominance, Iyer [21] explored the idea of extending dominance-based decision-making to problems with noisy evaluations. The author's idea was to eliminate alternatives which are dominated by another alternative according to the multi-criteria evaluations, without assuming that the aggregation method was known.

Much work has been carried out for the MAVT/MAUT with incomplete information which includes ordinal information as a particular case. Sage and White [39] proposed the model of imprecisely specified multiattribute utility theory (ISMAUT), in which precise preference information about both weights and utilities is not assumed. Malakooti [30] suggested a new algorithm for ranking and screening alternatives when there is incomplete information about the preferences and the value of the alternatives. An extended version of Malakooti's work was presented by Ahn [1]. Park, Kim, and colleagues [15,29,35] provided linear programming characterizations of dominance 
and potential optimality for decision alternatives when information about values and/or weights is not complete, extended the approach to hierarchical structures [29], and developed the concepts of weak potential optimality and strong potential optimality [35]. White and Holloway [47] considered an interactive selection process: a facilitator asks a decision maker questions and obtains responses that will be used to decide on the next question, with the eventual aim of identifying the most preferred alternative.

Finally, some methods follow decision rules to rank alternatives in situations with incomplete information about the weights. Barron and Barret [4] studied algebraic formulas such as equal weights and the use of ROC (rank order centroid) weights to select a representative weights vector $w$ from a set of admissible weights $W$, with the purpose of using $w$ to evaluate the alternatives. These authors concluded that ROC weights provide a better approximation than the other weighting vectors they considered. Other rules that have been proposed imply solving optimization problems [40]: the maximin rule consists of evaluating each alternative for its minimum (worst case) value; the minimax regret rule consists of evaluating each alternative for the maximum loss of value relative to a better alternative (the "maximum regret"); the central values rule consists of evaluating each alternative for the midpoint of the range of possible values. Although none of these rules ensures that the alternative indicated is the best and is the same that would be given if precise values for weights were elicited, simulations show that in general the alternative selected is among the best ones (e.g., [42]).

The work described in this paper relates to this last group of approaches which use rules based on information that is easy to elicit. Our objective is to rank the alternatives, or to select one alternative, without requiring precise information from the decision maker. We propose two new rules based on the ideas of the ROC weights rule that can deal with incomplete information on the value of each alternative in each criterion.

\section{Notation and decision rules}

\subsection{Notation}

Let us denote by $A=\left\{a_{1}, \ldots, a_{m}\right\}$ a discrete set of $m$ alternatives. Let $X=\left\{x_{1}, \ldots, x_{n}\right\}$ denote a set of $n$ criteria (attributes) for evaluating these alternatives. Let $v_{i}$ (.) denote the value function (or utility function-the difference here is not important) corresponding to criterion $x_{i}$. Consequently, $v_{i}\left(a_{j}\right) \in[0,1]$ denotes the value of the alternative $a_{j}$ according to criterion $x_{i}$.

According to the additive aggregation model, the global (multi-attribute) value of an alternative $a_{j} \in A$ is

$$
v\left(a_{j}\right)=\sum_{i=1}^{n} w_{i} v_{i}\left(a_{j}\right)
$$

where $w_{i}$ represents the scale coefficient or "weight" associated with $v_{i}$. For these parameters we have

$w_{1}, \ldots, w_{n} \geq 0$ and $\sum_{i=1}^{n} w_{i}=1$.

Without loss of generality we will consider that criteria weights are indexed in descending order, given the ordinal information provided by a decision maker, for instance comparing "swings" from the worst to the best performance in each value function $[14,44]$ (we assume the worst level is 0 and the best level is 1 in terms of value). Thus, the set of all vectors of weights compatible with this information is

$W=\left\{\left(w_{1}, w_{2}, \ldots w_{n}\right): w_{1} \geq w_{2} \geq \cdots \geq w_{n} \geq 0, \sum_{i=1}^{n} w_{i}=1\right\}$.

Let $V$ denote the set of the $n \times m$ matrices, whose elements are all values $v_{i}\left(a_{j}\right)(i=1, \ldots, n ; j=1, \ldots, m)$ compatible with the ordinal information provided by the decision maker. We will consider that we have a ranking of the value of each alternative in each criterion, and possibly also a ranking of the difference of values between consecutive alternatives in each criterion.

\subsection{Decision rules}

\subsubsection{Ordinal information on the weights}

Since criteria weights are usually the hardest parameters to elicit [38], research efforts have mostly focused on the case in which incomplete information only relates to the weights. Past studies have shown that some decision rules based on ordinal information about the weights lead to good results [2,40-42]. In [42], among other experiments, a set of Monte-Carlo simulations was performed in order to see how different rules (ROC weights, maximin rule, minimax regret rule and central values rule) compared for different indicators. The rules were compared, for example, in terms of their "hit rate", which indicates how often the alternative chosen with a vector of supposedly true weights (i.e., the vector that would be obtained if precise weights were elicited) coincides with the alternative indicated by the rule. The results indicate that, given a ranking of the weights, the ROC weights are the best rule (with a hit rate between $79 \%$ and $88 \%$, for problem sizes similar to those considered in this paper).

This work also used ROC weights when the ordinal information related to the weights. ROC weights are computed from the vertices of polytope $W$ (3). This polytope corresponds to a simplex whose vertices are $(1,0, \ldots, 0),\left(\frac{1}{2}, \frac{1}{2}, 0, \ldots, 0\right)$, $\left(\frac{1}{3}, \frac{1}{3}, \frac{1}{3}, 0, \ldots, 0\right), \ldots,(1 / n, 1 / n, \ldots, 1 / n)$. The coordinates of the centroid are found by averaging the corresponding coordinates of the defining vertices. ROC weights can be easily calculated using the following formula (recall that the indices of criteria reflect their order, $w_{1}$ is the highest weight and $w_{n}$ is the lowest one):

$w_{i}^{(R O C)}=\frac{1}{n} \sum_{j=i}^{n} \frac{1}{j}, i=1, \ldots, n$.

As reported by Butler and Olson [9], if ties exist extreme points will coincide. Only one of the tied values is included when obtaining the centroid. For instance, if there are three objectives and the decision maker states that $w_{1} \geq w_{2}$ and $w_{2}=w_{3}$, then the vertices are $(1,0,0)$, and $\left(\frac{1}{3}, \frac{1}{3}, \frac{1}{3}\right)$, hence $w_{1}=\frac{1}{2}\left(1+\frac{1}{3}\right)=\frac{2}{3}$ and $w_{2}=w_{3}=\frac{1}{2}\left(0+\frac{1}{3}\right)=\frac{1}{6}$. Solymosi and Dombi [43] described the process of generalizing the centroid approach to cases that include weak orders or partial orders.

\subsubsection{Ordinal information on the value of each alternative and value differences in each attribute}

If the decision maker states that it is difficult to indicate the exact value of each alternative in each attribute, a natural idea is to ask him for a ranking, e.g. "considering the attribute $x_{1}$, alternative $a_{1}$ is the best, followed by $a_{2}$ as the second best, and then $a_{3}$ ". In this work, we assume that for each attribute the worst level takes the value 0 and the best level takes the value 1 . This is a usual convention that is legitimate if the weights are set taking these levels into account.

As with ordinal information on the weights, we deem that in this case it is also possible to use an algebraic formula to choose a vector of values for each attribute that can approximately represent all the vectors' values compatible with the ordinal 
information. One possibility is to use ROC values for each attribute, i.e., the centroid of the polytope defined by the ranking of the values for that attribute. Since attributes are normalized such that the highest value for each attribute is 1 and the lowest value is 0 , the centroid corresponds to equally spaced values in the interval $[0,1]$. Hence, for attribute $x_{i}$, the ROC values are defined as follows $(i=1, \ldots, n)$ :

$v_{i}^{(R O C)}\left(a_{j}\right)=\frac{m-r_{i}\left(a_{j}\right)}{m-1}, \quad j=1, \ldots, m$,

where $r_{i}\left(a_{j}\right)$ represents the rank position of alternative $a_{j}$ considering the attribute $x_{i}$ and $r_{i}\left(a_{j}\right)<r_{i}\left(a_{k}\right) \Rightarrow v_{i}\left(a_{j}\right) \geq v_{i}\left(a_{k}\right)$. Suppose, for example, that we have 5 alternatives, and for criterion $x_{i}, \quad 1=v_{i}\left(a_{1}\right) \geq v_{i}\left(a_{2}\right) \geq v_{i}\left(a_{3}\right) \geq v_{i}\left(a_{4}\right) \geq v_{i}\left(a_{5}\right)=0$. The centroid of this simplex is $\left(1, \frac{3}{4}, \frac{2}{4}, \frac{1}{4}, 0\right)$ since the vertices are $(1,1,1,1,0),(1,1,1,0,0),(1,1,0,0,0)$ and $(1,0,0,0,0)$. In an example with $m$ alternatives, the centroid of the simplex defined by $1=v_{i}\left(a_{1}\right) \geq v_{i}\left(a_{2}\right) \geq \cdots \geq v_{i}\left(a_{m}\right)=0$ is equal to $(1,(m-2) /(m-1), \ldots, 1 /(m-1), 0)$.

The formula that approximates the values using ROC values can also be used if there are ties concerning the value of the alternatives in some criteria. Where there is one tie in one criterion, the problem is solved by decreasing the number of alternatives of the problem by one dimension, i.e, considering $m-1$ value levels instead of $m$ in that criterion. In cases with two ties the problem is solved by decreasing the problem by two dimensions, and so on. We assume that there is no attribute for which all alternatives have the same value (in practice such an attribute could be discarded).

In order to obtain better information about the alternatives' values, besides a ranking of the alternatives in each attribute it is also possible to ask the decision maker to provide a ranking of the differences between the values of consecutive alternatives. Suppose for instance that considering an attribute $x_{i}$, a decision maker outlines his rough idea of the relative position of 4 alternatives $a_{1}, a_{2}, a_{3}, a_{4}$ concerning their value according to $x_{i}$ as depicted in Fig. 1. From this outline we could ask the decision maker to confirm that not only $v_{i}\left(a_{4}\right) \geq v_{i}\left(a_{3}\right) \geq v_{i}\left(a_{2}\right) \geq v_{i}\left(a_{1}\right)$, but also to confirm that the ranking of the consecutive value differences $\Delta_{i 1}=v_{i}\left(a_{2}\right)-v_{i}\left(a_{1}\right), \quad \Delta_{i 2}=v_{i}\left(a_{3}\right)-v_{i}\left(a_{2}\right), \quad \Delta_{i 3}=v_{i}\left(a_{4}\right)-$ $v_{i}\left(a_{3}\right)$ is $\Delta_{i 1} \geq \Delta_{i 3} \geq \Delta_{i 2}$. This type of drawing-based elicitation has previously been proposed to obtain parameter values, for instance in [36], but in our case the objective is not to read an exact value for each alternative: we only consider ordinal information about the position of alternatives and about the difference of value between consecutive alternatives. Of course, the decision maker might also be asked directly about the rankorder of the consecutive value differences without using a graphical representation.

We can use this type of information to propose a rule to approximate the alternatives values. We will call this the $\triangle R O C$ rule. Let us consider an attribute $x_{i}$ and let us denote $s$ the number of different value levels in this attribute implied by the ordinal information provided by the decision maker, including the value levels $l_{i 1}=0$ and $l_{i s}=1$, which bound all other levels. For instance, if the ordinal information is $v_{i}\left(a_{4}\right) \geq v_{i}\left(a_{2}\right) \geq v_{i}\left(a_{1}\right) \geq v_{i}\left(a_{3}\right)$, then there are four different levels, which are by decreasing order $l_{i 4}=v_{i}\left(a_{4}\right)=1, l_{i 3}=v_{i}\left(a_{2}\right), l_{i 2}=v_{i}\left(a_{1}\right)$ and $l_{i 1}=v_{i}\left(a_{3}\right)=0$. Let us denote the value difference between two consecutive levels as $\Delta_{i k}=$ $l_{i(k+1)}-l_{i k}$, for $k=1, \ldots, s-1$. From these definitions, it is easy to check that:

$\Delta_{i 1}, \ldots, \Delta_{i(s-1)} \geq 0$ and $\sum_{k=1}^{s-1} \Delta_{i k}=1$.

Since the value differences are ranked, are positive, and add up to 1 , an expression similar to the formula used to derive ROC

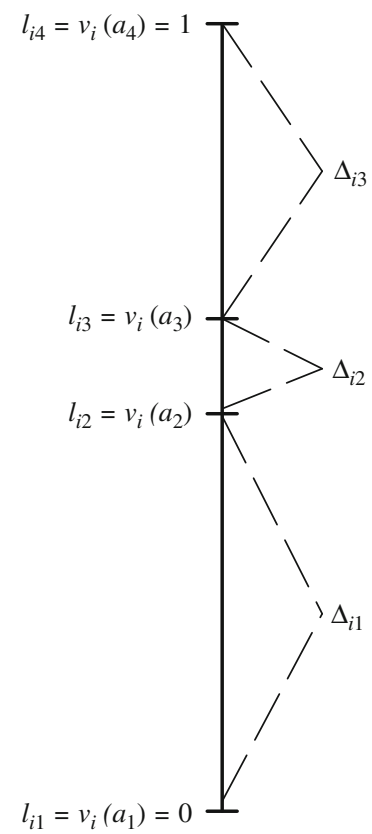

Fig. 1. Example of a rough drawing on a scale for attribute $x_{i}$.

weights can be used. The approximation to the values of each alternative in each criterion can be obtained using the following algorithm:

Step 1: Ask the decision maker to provide a ranking of the alternatives in each criterion $x_{i}$ (possibly with ties). Label the resulting different levels as $l_{i 1}, \ldots, l_{i s}$, ranked from lowest to highest, with $l_{i 1}=0$ and $l_{i s}=1$. Each level will correspond to the value of one alternative (or more than one, if there are ties).

Step 2: Ask the decision maker to provide a ranking of the difference of values between consecutive levels $\Delta_{i 1}, \ldots, \Delta_{i(s-1)}$. For each $k=1, \ldots, s-1$, let $\operatorname{rank}(k)$ denote the rank of $\Delta_{i k}$ within the set $\left\{\Delta_{i 1}, \ldots, \Delta_{i(s-1)}\right\}$. This rank is an integer between 1 and $s-1$, with rank 1 denoting the highest difference.

Step 3: Determine a rank order centroid for $s-1$ variables:

$\Delta_{[k]}=\frac{1}{s-1} \sum_{j=k}^{s-1} \frac{1}{j}, \quad k=1, \ldots, s-1$.

Step 4: For each $k=1, \ldots, s-1$, set the values provided by the centroid approximation: $\Delta_{i k}^{(R O C)}=\Delta_{[\operatorname{rank}(k)]}$.

Step 5: The approximate values for the levels in attribute $x_{i}$, are then defined as follows:

$l_{i 1}=0$,

$l_{i j}=\sum_{k=1}^{j-1} \Delta_{i k}^{(R O C)}, \quad j=2, \ldots, S$.

Step 6: $v_{i}^{(\triangle R O C)}\left(a_{j}\right)$, the approximate values in attribute $x_{i}$ for alternative $a_{j}(j=1, \ldots, m)$ based on the $\triangle R O C$ values rule, is equal to the approximate value of the respective level, according to the values for the level correspondence created in step 1.

For the example presented in Fig. 1, the ROC for three differences of value (as there are 4 alternatives) is $\Delta_{[1]}=\frac{11}{18}$, $\Delta_{[2]}=\frac{5}{18}$, and $\Delta_{[3]}=\frac{2}{18}$. Taking into account the order $\Delta_{i 1} \geq \Delta_{i 3} \geq \Delta_{i 2}$, we obtain $\Delta_{i 1}^{(R O C)}=\frac{11}{18}, \Delta_{i 2}^{(R O C)}=\frac{2}{18}$, and $\Delta_{i 3}^{(R O C)}=\frac{5}{18}$. Therefore, the $\triangle R O C$ values vector is $\left(0, \frac{11}{18}, \frac{13}{18}, 1\right)$ :

- $v_{i}^{(\Delta R O C)}\left(a_{1}\right)=0$,

- $v_{i}^{(\Delta R O C)}\left(a_{2}\right)=\Delta_{i 1}^{(R O C)}=\frac{11}{18}$, 
- $v_{i}^{(\triangle R O C)}\left(a_{3}\right)=\Delta_{i 1}^{(R O C)}+\Delta_{i 2}^{(R O C)}=\frac{11}{18}+\frac{2}{18}=\frac{13}{18}$,

- $v_{i}^{(\Delta R O C)}\left(a_{4}\right)=\Delta_{i 1}^{(R O C)}+\Delta_{i 2}^{(R O C)}+\Delta_{i 3}^{(R O C)}=\frac{11}{18}+\frac{2}{18}+\frac{5}{18}=1$.

Note that this algorithm can account for the existence of ties in the value of the alternatives in each criterion. Ties in the ranking of consecutive value differences in step 2, on the other hand, can be dealt with using the procedures proposed for ties in the criteria weights $[9,43]$.

\section{Simulation}

In the previous section we presented simple rules that can be used to generate approximate values for the weights and for the alternatives' values in each attribute, given the ordinal information about these parameters. The parameter values derived by these rules can then be used to select a promising subset of alternatives. The decision maker should nonetheless have an idea of how good these proposed alternatives are compared with the results that would have been achieved if the precise cardinal values for all the parameters had been elicited (assuming such a precise elicitation was possible). In this section we describe a sequence of experiments using Monte Carlo simulation to compare the results provided by rules with the results that are obtained for precise cardinal information.

Each generated random problem is characterized by a (precise) value matrix and a (precise) weights vector. Applying the additive model to the random data generated we then compute the overall value of each alternative and obtain the corresponding ranking of the alternatives. This is what we call the supposedly true ranking, i.e. the ranking that would be obtained if cardinal information was elicited. Each of the rules is applied considering only the ordinal information contained in the data generated. Using the approximate parameter values derived from a rule we can build the ranking of the alternatives that the rule yields. Comparing the ranking of the alternatives according to the supposedly true parameters with the ranking of the alternatives according to a decision rule, we may consider the following results:

- The position that the best alternative according to the true ranking reaches in the ranking generated by the decision rule: this allows us to know how many alternatives from the top of the ranking provided by the rule must be kept so as to include the supposedly best alternative in the set of selected alternatives.

- The position that the best alternative in the ranking generated by the rule reaches in the supposedly true ranking: this allows us to know how good the alternative chosen by the rule is in terms of the supposedly true ranking.

Like Barron and Barret [4] we also calculated the "value loss", i.e. the multiattribute value difference between the alternative selected by a decision rule and the true best alternative, considering the supposedly true parameter values.

In these experiments we considered situations with 5,10 , and 15 attributes, and 5, 10, and 15 alternatives. Like [40] and [42], we generated 5000 random problems for each problem dimension (after verifying that using a greater number of problems did not significantly affect the results). The uniform distribution was considered for all the parameters generated, as in most comparable previous experiments $([2,40,42])$.

The scaling weights were generated on the basis of a uniform distribution in $W$ using the process described in [8]. To obtain weights for $n$ attributes, we generate $n-1$ independent random numbers from a uniform distribution on $[0,1]$ and rank these numbers. Let the ranked numbers be $r_{(n-1)} \geq \cdots \geq r_{(2)} \geq r_{(1)}$. The following differences can then be obtained: $w_{n}=1-r_{(n-1)}$, $w_{n-1}=r_{(n-1)}-r_{(n-2)}, \ldots, w_{1}=r_{(1)}-0$. Then the set of numbers $\left(w_{1}, w_{2}, \ldots, w_{n}\right)$ adds up to 1 and is uniformly distributed on the simplex defined by the rank-order constraints (3), after relabelling.

The single-attribute values $v_{i}\left(a_{j}\right)$ were generated from a uniform distribution in the interval $[0,1]$ and then normalized attribute-wise in such a way that the highest value in each attribute is 1 and the lowest value is 0 . For each criterion, let $v_{i}^{l o}$ and $v_{i}^{h i}$ denote the lowest and highest values in the $m$ values generated. Then the normalized value of $v_{i}\left(a_{j}\right)$ is equal to $\left(v_{i}\left(a_{j}\right)-v_{i}^{l o}\right) /\left(v_{i}^{h i}-v_{i}^{l o}\right)$.

The first simulations were performed considering known cardinal weights and ordinal information on the values (with and without ordinal information on the value differences). Additional simulations were performed in which ordinal information on weights and ordinal information on the values (again, with and without ordinal information on the value differences) where considered simultaneously. The situation with known cardinal values and ordinal information on the weights has already been studied in [42]. Results are presented in the next section.

\section{Results}

5.1. Incomplete information on the value of each alternative in each attribute

In this set of experiments we considered that the precise weights of the criteria (henceforth referred to as TRUE weights) were known, but we supposed that the decision maker indicated incomplete information about the value of each alternative in each criterion. We tested the ROC values rule (assuming that the decision maker ranked the alternatives) and the $\triangle R O C$ values rule (assuming that the decision maker ranked the alternatives and also ranked the difference between consecutive alternatives) to derive approximate values for the alternatives in each criterion.

A first set of experiments was carried out in order to see how the different rules compare if the analysis aims at selecting only the best alternative according to a rule. These experiments indicated the position reached by the alternative suggested by the ROC values and $\triangle R O C$ values rule on the supposedly true ranking. Detailed results are presented in tabular form in Table 1 (in this table TRUE $\triangle R O C$ indicates the use of TRUE weights and $\triangle R O C$ values and TRUE ROC indicates the use of TRUE weights and ROC values). This table shows, for each rule and for each size, the average position in the supposedly true ranking (the minimum position was always 1) and the percentage of cases where the position reached is $1,2,3,4$ or higher. Note that the percentage of cases where the position reached is equal to 1 is the hit rate.

The results indicate that the use of supposedly TRUE weights and $\triangle R O C$ values leads to a hit rate of at least $90 \%$. It can also be seen that the hit rate increases with the number of alternatives. Using TRUE weights and ROC values the hit rate varies between $76 \%$ and $81 \%$. Since the use of the $\triangle R O C$ rule requires more information than the use of the ROC rule it is not surprising that the former performs better. But it is noteworthy that the use of $\triangle R O C$ values leads to a considerable increase in the hit rate compared with the use of ROC values.

Instead of using a rule to select a single alternative, the analysis can aim at retaining a small subset of promising alternatives for further analysis, in a strategy of progressive reduction of the number of candidates. In such cases, the objective is to retain as small a subset of alternatives as possible, yet 
Table 1

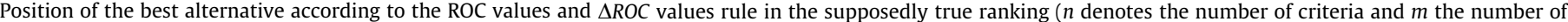
alternatives).

\begin{tabular}{|c|c|c|c|c|c|c|c|c|c|c|c|c|c|}
\hline \multirow[t]{2}{*}{$n$} & \multirow[t]{2}{*}{$m$} & \multicolumn{6}{|c|}{ TRUE $\triangle R O C$} & \multicolumn{6}{|c|}{ TRUE ROC } \\
\hline & & Average & $\% 1$ & $\% 2$ & $\% 3$ & $\% 4$ & $\% \geq 5$ & Average & $\% 1$ & $\% 2$ & $\% 3$ & $\% 4$ & $\% \geq 5$ \\
\hline 5 & 5 & 1.10 & 91.00 & 8.20 & 0.72 & 0.08 & 0.00 & 1.25 & 78.78 & 17.44 & 3.36 & 0.40 & 0.02 \\
\hline 5 & 10 & 1.09 & 91.82 & 7.36 & 0.68 & 0.14 & 0.00 & 1.28 & 78.66 & 16.38 & 3.64 & 1.04 & 0.28 \\
\hline 5 & 15 & 1.08 & 93.32 & 5.88 & 0.62 & 0.18 & 0.00 & 1.29 & 79.02 & 15.30 & 4.14 & 1.06 & 0.40 \\
\hline 10 & 5 & 1.10 & 90.76 & 8.46 & 0.74 & 0.04 & 0.00 & 1.24 & 80.94 & 14.92 & 3.38 & 0.70 & 0.06 \\
\hline 10 & 10 & 1.09 & 91.58 & 7.54 & 0.76 & 0.12 & 0.00 & 1.32 & 77.38 & 16.02 & 4.50 & 1.58 & 0.52 \\
\hline 10 & 15 & 1.08 & 93.00 & 6.28 & 0.64 & 0.08 & 0.00 & 1.31 & 77.76 & 16.04 & 4.50 & 1.16 & 0.54 \\
\hline 15 & 5 & 1.10 & 90.80 & 8.36 & 0.80 & 0.04 & 0.00 & 1.26 & 79.20 & 16.64 & 3.38 & 0.70 & 0.08 \\
\hline 15 & 10 & 1.09 & 91.88 & 7.12 & 0.92 & 0.06 & 0.02 & 1.31 & 76.84 & 16.7 & 4.64 & 1.26 & 0.56 \\
\hline 15 & 15 & 1.08 & 92.80 & 6.40 & 0.64 & 0.14 & 0.00 & 1.30 & 79.00 & 14.74 & 4.40 & 1.44 & 0.42 \\
\hline
\end{tabular}

Table 2

Position of the supposedly best alternative in the ranking induced by the ROC values and $\triangle R O C$ values rule.

\begin{tabular}{|c|c|c|c|c|c|c|c|c|c|c|c|c|c|}
\hline \multirow[t]{2}{*}{$n$} & \multirow[t]{2}{*}{$m$} & \multicolumn{6}{|c|}{ TRUE $\triangle R O C$} & \multicolumn{6}{|c|}{ TRUE ROC } \\
\hline & & Average & $\% 1$ & $\% 2$ & $\% 3$ & $\% 4$ & $\% \geq 5$ & Average & $\% 1$ & $\% 2$ & $\% 3$ & $\% 4$ & $\% \geq 5$ \\
\hline 5 & 5 & 1.10 & 91.00 & 8.12 & 0.80 & 0.08 & 0.00 & 1.25 & 78.78 & 17.60 & 3.28 & 0.30 & 0.04 \\
\hline 5 & 10 & 1.09 & 91.82 & 7.50 & 0.64 & 0.04 & 0.00 & 1.28 & 78.66 & 16.44 & 3.68 & 0.94 & 0.28 \\
\hline 5 & 15 & 1.07 & 93.32 & 5.96 & 0.66 & 0.04 & 0.02 & 1.29 & 79.02 & 14.92 & 4.40 & 1.20 & 0.30 \\
\hline 10 & 5 & 1.10 & 90.76 & 8.46 & 0.76 & 0.02 & 0.00 & 1.24 & 80.94 & 15.10 & 3.22 & 0.64 & 0.01 \\
\hline 10 & 10 & 1.10 & 91.58 & 7.26 & 1.10 & 0.04 & 0.02 & 1.32 & 77.38 & 16.06 & 4.80 & 1.24 & 0.52 \\
\hline 10 & 15 & 1.08 & 93.00 & 6.26 & 0.72 & 0.02 & 0.00 & 1.31 & 77.76 & 16.36 & 3.72 & 1.42 & 0.74 \\
\hline 15 & 5 & 1.10 & 90.80 & 8.38 & 0.76 & 0.06 & 0.00 & 1.26 & 79.20 & 16.56 & 3.40 & 0.80 & 0.04 \\
\hline 15 & 10 & 1.09 & 91.88 & 7.10 & 0.90 & 0.06 & 0.00 & 1.31 & 76.84 & 16.82 & 4.44 & 1.36 & 0.54 \\
\hline 15 & 15 & 1.08 & 92.80 & 6.48 & 0.64 & 0.06 & 0.02 & 1.29 & 79.00 & 14.88 & 4.58 & 1.12 & 0.42 \\
\hline
\end{tabular}

without eliminating the best one. An interesting question is to know how many alternatives should be retained when a rule based on ordinal information is used. To answer this question our simulations studied the position of the supposedly best alternative in the ranking produced by each rule. Table 2 presents, for each problem size, the average position of the supposedly best alternative in the ranking provided by each rule (the minimum position was always 1 ), as well as the percentage of cases where the position is $1,2,3,4$, or higher. The probability of retaining the supposedly best alternative increases naturally with the number of alternatives that are retained. In all cases, selecting two alternatives would suffice to keep the supposedly best one in $93 \%$ of the cases, while selecting three alternatives would suffice in $97 \%$ of the cases. We can see that the additional information required from the decision maker by the $\triangle R O C$ values rule is rewarded by clearly superior results compared with the ROC values rule. Indeed, in at least $99 \%$ of the cases the supposedly best alternative was one of the two best ranked alternatives according to the $\triangle R O C$ values rule.

In Table 3 it is possible to see the value loss implied by selecting the top-ranked alternative obtained by the different rules. In this table ROC TRUE refers to the use of ROC weights and TRUE values, ROC $\triangle R O C$ refers to the use of ROC weights and $\triangle R O C$ values, ROC ROC refers to the use of ROC weights and ROC values, TRUE $\triangle R O C$ refers to the use of TRUE weights and $\triangle R O C$ values, and TRUE ROC refers to the use of TRUE weights and ROC values. Assuming the weights are known and using $\triangle R O C$ values (TRUE $\triangle R O C$ columns), the average value loss varies between 0.0070 and 0.0316 . The maximum value loss is a value between 0.0580 and 0.2455 . Assuming the weights are known and using ROC values (TRUE ROC columns), the average value loss varies between 0.0051 and 0.0139 . The maximum value loss is a value between 0.0998 and 0.3123 . On average, when taking the weights as known, using the $\triangle R O C$ values leads to roughly half the loss of value incurred by using the ROC values. Note, however, that in both cases the average value loss can be considered very small.

\subsection{Incomplete information on weights and the value of each alternative in each attribute}

In this section we consider that the criteria weights and the value of each alternative in each criterion are unknown. The decision maker indicates only ordinal information about the weights and about the value of alternatives in each criterion, possibly adding ordinal information about differences of value between consecutive alternatives in each criterion. We tested the rule of using ROC weights together with ROC values, as well as the rule of using ROC weights together with $\triangle R O C$ values. Table 4 presents the position in the supposedly true ranking of the best alternative obtained by each rule (in this table ROC $\triangle R O C$ means the use of ROC weights and $\triangle R O C$ values and ROC ROC means the use of ROC weights and ROC values). Using ROC weights and $\triangle R O C$ values the hit rate (column \%1) decreases with the number of alternatives.

Table 5 presents the position of the supposedly true alternative in the ranking induced by the ROC weights/ROC values and ROC weights $\triangle \triangle R O C$ values rule. In the previous experiments, which considered TRUE weights and $\triangle R O C$ values, the results indicated the hit rate was higher than $90 \%$. If we consider that we also do not know the weights, and use ROC weights, the results are still fairly good (the hit rate is greater than $78 \%$ ). If the two top-ranked alternatives obtained using ROC weights and $\triangle R O C$ values are 
Table 3

Value loss implied by selecting the best alternative according to a rule based on ordinal information.

\begin{tabular}{|c|c|c|c|c|c|c|c|c|c|c|c|}
\hline \multirow[t]{2}{*}{$n$} & \multirow[t]{2}{*}{$m$} & \multicolumn{2}{|c|}{ ROC TRUE } & \multicolumn{2}{|c|}{$\mathrm{ROC} \triangle R O C$} & \multicolumn{2}{|l|}{ ROC ROC } & \multicolumn{2}{|c|}{ TRUE $\triangle R O C$} & \multicolumn{2}{|c|}{ TRUE ROC } \\
\hline & & Average & Maximum & Average & Maximum & Average & Maximum & Average & Maximum & Average & Maximum \\
\hline 5 & 5 & 0.0589 & 0.4222 & 0.0663 & 0.4926 & 0.0816 & 0.5351 & 0.0316 & 0.2455 & 0.0655 & 0.3123 \\
\hline 5 & 10 & 0.0433 & 0.2999 & 0.0482 & 0.3177 & 0.0668 & 0.5143 & 0.0171 & 0.0994 & 0.0459 & 0.2377 \\
\hline 5 & 15 & 0.0383 & 0.3009 & 0.0400 & 0.2364 & 0.0543 & 0.4223 & 0.0076 & 0.1755 & 0.0391 & 0.2105 \\
\hline 10 & 5 & 0.0391 & 0.3053 & 0.0459 & 0.3605 & 0.0662 & 0.3808 & 0.0238 & 0.1195 & 0.0535 & 0.2602 \\
\hline 10 & 10 & 0.0314 & 0.3092 & 0.0339 & 0.3466 & 0.0480 & 0.2845 & 0.0119 & 0.0705 & 0.0367 & 0.1996 \\
\hline 10 & 15 & 0.0276 & 0.2158 & 0.0292 & 0.2780 & 0.0412 & 0.2804 & 0.0100 & 0.0607 & 0.0292 & 0.1599 \\
\hline 15 & 5 & 0.0285 & 0.2217 & 0.0365 & 0.4018 & 0.0534 & 0.3167 & 0.0207 & 0.0991 & 0.0447 & 0.2341 \\
\hline 15 & 10 & 0.0236 & 0.2448 & 0.0280 & 0.1751 & 0.0346 & 0.1946 & 0.0111 & 0.0693 & 0.0311 & 0.1922 \\
\hline 15 & 15 & 0.0207 & 0.1610 & 0.0215 & 0.1869 & 0.0355 & 0.2080 & 0.0070 & 0.0580 & 0.0243 & 0.0998 \\
\hline
\end{tabular}

Table 4

Position of the best alternative according to the ROC weights/ROC values and ROC weights/ $\triangle R O C$ values rule in the supposedly true ranking.

\begin{tabular}{|c|c|c|c|c|c|c|c|c|c|c|c|c|c|}
\hline \multirow[t]{2}{*}{$n$} & \multirow[t]{2}{*}{$m$} & \multicolumn{6}{|c|}{$\mathrm{ROC} \triangle R O C$} & \multicolumn{6}{|l|}{ ROC ROC } \\
\hline & & Average & $\% 1$ & $\% 2$ & $\% 3$ & $\% 4$ & $\% \geq 5$ & Average & $\% 1$ & $\% 2$ & $\% 3$ & $\% 4$ & $\% \geq 5$ \\
\hline 5 & 5 & 1.21 & 83.12 & 13.40 & 3.06 & 0.42 & 0.00 & 1.32 & 74.98 & 19.22 & 4.74 & 0.98 & 0.08 \\
\hline 5 & 10 & 1.29 & 79.44 & 14.84 & 3.88 & 1.42 & 0.42 & 1.41 & 72.92 & 17.80 & 6.00 & 2.42 & 0.90 \\
\hline 5 & 15 & 1.31 & 78.98 & 14.56 & 4.18 & 1.34 & 0.60 & 1.47 & 71.26 & 17.86 & 6.52 & 2.70 & 0.92 \\
\hline 10 & 5 & 1.21 & 83.02 & 13.90 & 2.66 & 0.38 & 0.04 & 1.31 & 75.98 & 18.14 & 4.62 & 1.18 & 0.08 \\
\hline 10 & 10 & 1.25 & 81.42 & 13.82 & 3.42 & 1.02 & 0.32 & 1.42 & 72.26 & 18.24 & 60.6 & 2.26 & 1.18 \\
\hline 10 & 15 & 1.29 & 80.50 & 13.90 & 3.48 & 1.20 & 0.92 & 1.46 & 71.84 & 17.92 & 6.08 & 2.36 & 1.80 \\
\hline 15 & 5 & 1.19 & 84.40 & 12.44 & 2.76 & 0.40 & 0.00 & 1.32 & 76.20 & 17.52 & 4.92 & 1.12 & 0.24 \\
\hline 15 & 10 & 1.23 & 83.24 & 12.72 & 2.74 & 0.94 & 0.36 & 1.39 & 74.02 & 17.40 & 5.48 & 2.12 & 0.98 \\
\hline 15 & 15 & 1.26 & 81.40 & 13.30 & 3.76 & 1.02 & 0.52 & 1.39 & 75.22 & 15.80 & 5.74 & 1.86 & 1.38 \\
\hline
\end{tabular}

Table 5

Position of the supposedly best alternative in the ranking induced by the ROC weights/ROC values and ROC weights/ $\triangle R O C$ values rule.

\begin{tabular}{|c|c|c|c|c|c|c|c|c|c|c|c|c|c|}
\hline \multirow[t]{2}{*}{$n$} & \multirow[t]{2}{*}{$m$} & \multicolumn{6}{|c|}{$\mathrm{ROC} \triangle R O C$} & \multicolumn{6}{|l|}{ ROC ROC } \\
\hline & & Average & $\% 1$ & $\% 2$ & $\% 3$ & $\% 4$ & $\% \geq 5$ & Average & $\% 1$ & $\% 2$ & $\% 3$ & $\% 4$ & $\% \geq 5$ \\
\hline 5 & 5 & 1.21 & 83.12 & 13.54 & 2.90 & 0.44 & 0.00 & 1.32 & 75.00 & 19.28 & 4.46 & 1.10 & 0.16 \\
\hline 5 & 10 & 1.28 & 79.44 & 15.16 & 3.70 & 1.14 & 0.56 & 1.43 & 72.92 & 17.84 & 5.46 & 2.18 & 1.60 \\
\hline 5 & 15 & 1.31 & 78.98 & 14.66 & 4.08 & 1.28 & 0.68 & 1.47 & 71.26 & 17.84 & 6.66 & 2.48 & 1.08 \\
\hline 10 & 5 & 1.20 & 83.02 & 14.08 & 2.58 & 0.30 & 0.02 & 1.32 & 75.98 & 17.26 & 5.42 & 1.24 & 0.10 \\
\hline 10 & 10 & 1.25 & 81.42 & 13.66 & 3.50 & 1.10 & 0.32 & 1.43 & 72.26 & 18.26 & 5.86 & 2.36 & 1.26 \\
\hline 10 & 15 & 1.28 & 80.50 & 13.82 & 3.72 & 1.32 & 0.64 & 1.46 & 71.84 & 17.48 & 6.72 & 2.26 & 1.70 \\
\hline 15 & 5 & 1.19 & 84.40 & 12.60 & 2.54 & 0.42 & 0.04 & 1.31 & 76.20 & 18.18 & 4.42 & 1.04 & 0.16 \\
\hline 15 & 10 & 1.22 & 83.24 & 12.86 & 2.78 & 0.88 & 0.24 & 1.39 & 74.02 & 17.32 & 5.74 & 1.96 & 0.96 \\
\hline 15 & 15 & 1.25 & 81.40 & 13.54 & 3.76 & 0.90 & 0.40 & 1.38 & 75.22 & 16.22 & 5.20 & 2.44 & 0.92 \\
\hline
\end{tabular}

kept, instead of just one, then the supposedly best alternative is one of these two in at least $94 \%$ of the cases.

The results are obviously worse than in the case with known weights, as in this situation the rules use less information. Nevertheless, it should be noted that combining ROC weights with $\triangle R O C$ values yields results very close to those obtained in [42], assuming that the values of the alternatives were known (see Table 6). Once again the additional information requested from the decision maker by the $\triangle R O C$ values rule is rewarded by better results compared with the ROC values rule. These results suggest that using the ROC weights and $\triangle R O C$ values rule to facilitate the elicitation of information leads to a rapid identification of the most promising alternatives.
Table 3 presents the value loss of the different rules. Considering the weights to be unknown (using ROC weights) and using $\triangle R O C$ values the average value loss varies between 0.0215 and 0.0663 . The maximum value loss is between 0.1869 and 0.4926 . Considering the weights are unknown and using ROC values, the average value loss increases to between 0.0355 and 0.0816 , and the maximum value loss is between 0.1946 and 0.5351 . Considering both the weights and the values of each alternative in each attribute to be unknown, the average value loss is still small. Comparison of the third and fifth columns of Table 3 indicates that when there is only ordinal information about the weights and ROC weights are used the average loss of value using $\triangle R O C$ values is very similar to the average loss of 
Table 6

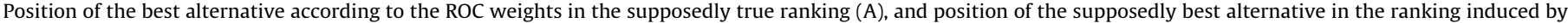
the ROC weights (B), assuming the true values for the alternatives were known (results from [42]).

\begin{tabular}{|c|c|c|c|c|c|c|c|c|c|c|c|c|c|}
\hline \multirow[t]{2}{*}{$n$} & \multirow[t]{2}{*}{$m$} & \multicolumn{6}{|l|}{ (A) } & \multicolumn{6}{|l|}{ (B) } \\
\hline & & Average & $\% 1$ & $\% 2$ & $\% 3$ & $\% 4$ & $\% \geq 5$ & Average & $\% 1$ & $\% 2$ & $\% 3$ & $\% 4$ & $\% \geq 5$ \\
\hline 5 & 5 & 1.18 & 84.96 & 12.26 & 2.40 & 0.34 & 0.04 & 1.18 & 84.96 & 12.16 & 2.66 & 0.22 & 0.00 \\
\hline 5 & 10 & 1.25 & 81.76 & 13.22 & 3.58 & 1.10 & 0.34 & 1.25 & 81.76 & 13.36 & 3.54 & 1.00 & 1.34 \\
\hline 5 & 15 & 1.30 & 79.72 & 14.14 & 3.90 & 1.36 & 0.88 & 1.29 & 79.72 & 14.40 & 3.90 & 1.24 & 0.74 \\
\hline 10 & 5 & 1.17 & 85.58 & 11.98 & 2.18 & 0.20 & 0.06 & 1.17 & 85.58 & 12.00 & 1.94 & 0.42 & 0.06 \\
\hline 10 & 10 & 1.24 & 82.46 & 13.10 & 3.20 & 0.78 & 0.46 & 1.24 & 82.46 & 12.76 & 3.36 & 1.02 & 0.40 \\
\hline 10 & 15 & 1.27 & 80.80 & 13.58 & 3.84 & 1.26 & 0.52 & 1.27 & 80.80 & 13.78 & 3.94 & 0.90 & 0.58 \\
\hline 15 & 5 & 1.14 & 87.92 & 10.08 & 1.76 & 0.24 & 0.00 & 1.15 & 87.92 & 9.92 & 1.82 & 0.26 & 0.04 \\
\hline 15 & 10 & 1.20 & 84.46 & 12.10 & 2.64 & 0.50 & 0.30 & 1.21 & 84.46 & 11.92 & 2.66 & 0.66 & 0.30 \\
\hline 15 & 15 & 1.24 & 82.96 & 12.34 & 3.30 & 1.08 & 0.32 & 1.23 & 82.96 & 12.94 & 2.74 & 0.88 & 0.48 \\
\hline
\end{tabular}

value implied by the ROC weights alone (i.e., considering TRUE values for the alternatives).

\section{Conclusions}

This work has presented a sequence of Monte-Carlo simulations with the aim of assessing different decision rules for the context where there is only ordinal information about the weights of the attributes and about the values of each alternative in each attribute. The rules studied in this paper involved computing approximate values for the parameters, which can then be used for ranking alternatives based on the multiattribute additive aggregation model.

Previous studies considered using rules of this type based on algebraic formulas to compute approximate values for the weights. If the decision maker provides a ranking of the weights, then the ROC weights rule was found to provide the best approximation [4], and was found to perform even better than more complex optimization-based rules [42]. The originality of our study lies in the consideration of analogous decision rules for the case in which we also have ordinal information about the value of each alternative in each attribute. We proposed an adaption of the ROC weights rule for this purpose: the ROC values rule. We also proposed a new rule which requires a little more information (but which is easily elicited, for example, by a rough drawing): the $\triangle R O C$ values rule.

Another noteworthy aspect of this study is that we tested strategies to select more than one alternative. This contrasts with the assumption that the decision maker uses these rules to select only the top alternative according to the rule, which would lead us to focus only on the calculation of hit rates and loss of value. Hence, we were also interested in finding out how many alternatives should be kept to ensure a good probability of not excluding the truly best one. The objective of this strategy is to simplify the problem in terms of the number of alternatives so as to study the most promising ones in more detail, or to elicit more information. This is particularly useful when assessing the performance of all alternatives under all the criteria would imply significant expense, time, or effort.

As our experiments have shown, using ordinal information generally leads to good results in the identification of the most promising alternatives. The best rule presented for cases without any cardinal information was the combined use of ROC weights and $\triangle R O C$ values. With this rule, the hit rate varies between $79 \%$ and $85 \%$. This rule is also very useful for selecting a subset of the most promising alternatives: selecting the two best alternatives according to this rule is sufficient in $94 \%$ of the cases or more, depending on the problem size, to retain the best alternative according to the true weights and values.

Another finding resulting from these experiments is that the $\triangle R O C$ values rule leads to results that are clearly superior to the results of the ROC values rule, with the average value loss reduced to roughly one half, and in some cases even less. Finally, we found out that the combined use of ROC weights and $\triangle R O C$ values does not provide results significantly worse than when ROC weights are used in a situation where the cardinal values are known.

The elicitation of ordinal information makes the cognitive task of the decision maker easier. Hence, given these results, we deem that the use of this type of rules to identify a small subset containing the most promising alternatives is an interesting possibility, whenever it is costly or difficult to obtain precise values for all parameters. Eliciting ordinal information about consecutive value differences requires a little additional effort, but the resulting increase in the quality of the results, in our opinion, justifies this extra step.

The conclusions presented in this paper should be read carefully, since the experiments were restricted to the case where the decision is based on a complete ranking of the criterion weights and on a complete ranking of the value of each alternative in each attribute. For the case where the set of acceptable weights and the set of acceptable values are defined by a set of general linear restrictions, it is possible that the ROC and $\triangle R O C$ rules lose some power. However, as referred by [4], the ROC is a specific example of centroid values, which generalizes to any convex value set specified by linear inequalities, and for a large class of situations the centroid computations are not very difficult [43] Testing the quality of centroid-based approximations for other types of constraints is an interesting subject for future research.

Another future research path is the use of this type of approximations in multi-actor settings, namely group decisions and negotiations, where eliciting order relations is less prone to disagreement than eliciting precise parameter values. Finally, we deem that this type of approximations might also be interesting to be studied for other multi-criteria methods besides additive MAVT.

\section{Acknowledgements}

This work benefited from support of FCT/FEDER Grant POCI/ EGE/58371/2004. P. Sarabando was supported by FCT under Grant SFRH/BD/29178/2006. 


\section{References}

[1] Ahn B. Extending Malakooti's model for ranking multicriteria alternatives with preference strength and partial information. IEEE Transactions on Systems Man and Cybernetics Part A: Systems and Humans 2003;33(3): 281-7.

[2] Ahn B, Park K. Comparing methods for multiattribute decision making with ordinal weights. Computers and Operations Research 2008;35:1660-70.

[3] Bana e Costa C, De Corte J, Vansnick J. On the mathematical foundation of MACBETH. In: Figueira J, Greco S, Ehrgott M, editors. Multiple criteria decision analysis: state of the art surveys. Boston, Dordrecht, London: Springer Verlag; 2005. p. 409-43.

[4] Barron F, Barrett B. Decision quality using ranked attribute weights. Management Science 1996;42(11):1515-23.

[5] Belton V, Stewart T. Multiple criteria decision analysis: an integrated approach. Dordrecht: Kluwer Academic; 2002.

[6] Bisdorff R. Concordant outranking with multiple criteria of ordinal significance-a contribution to robust multicriteria aid for decision. 4OR 2004;2:293-308.

[7] Bouyssou D, Pirlot M. Ordinal aggregation and strict preferences for multi-attributed alternatives, Technical Report, cahiers du LAMSADE, No. 212, 2003.

[8] Butler J, Jia J, Dyer J. Simulation techniques for the sensitivity analysis of multi-criteria decision models. European Journal of Operational Research 1997;103(3):531-46.

[9] Butler J, Olson D. Comparison of centroid and simulation approaches for selection sensitivity analysis. Journal of Multi-Criteria Decision Analysis 1999;8:146-61.

[10] Contreras I, Marmol A. A lexicographical compromise method for multiple criteria group decision problems with imprecise information. European Journal of Operational Research 2007;181(3):1530-9.

[11] Cook W, Kress M, Seiford L. A general framework for distance-based consensus in ordinal ranking model. European Journal of Operationa Research 1996;92:392-7.

[12] Dias L. A note on the role of robustness analysis in decision-aiding processes. In: Roy B, Aloulou M, Kalai R, editors. Robustness in OR-DA Lamsade; 2007. p. 53-70. annales du Lamsade no 7.

[13] Dias L, Clímaco J. Additive aggregation with variable interdependent parameters: the VIP analysis software. Journal of the Operational Research Society 2000;51(9):1070-82.

[14] Edwards W, Hutton Barron F. SMART and SMARTER: improved simple methods for multiattribute utility measurement. Organizational Behavior and Human Decision Processes 1994;60:306-25.

[15] Eum Y, Park K, Kim H. Establishing dominance and potential optimality in multi-criteria analysis with imprecise weights and value. Computers \& Operations Research 2001;28(5):397-409.

[16] Figueira J, Greco S, Ehrgott M, editors. Multiple criteria decision analysis: state of the art surveys. Boston, Dordrecht, London: Springer; 2005

[17] Figueira J, Greco S, Slowinski R. Building a set of additive value functions representing a reference preorder and intensities of preference: grip method. Technical Report, cahiers du LAMSADE, No. 253, 2007.

[18] Gonzalez-Pachon J, Romero C. Aggregation of partial ordinal rankings: an interval goal programming approach. Computers and Operations Research 2001;28(8):827-34

[19] Greco S, Matarazzo B, Slowinski R. Decision rule approach. In: Figueira J, Greco S, Ehrgott M, editors. Multiple criteria decision analysis: state of the art surveys. Boston, Dordrecht, London: Springer Verlag; 2005. p. 507-62.

[20] Hazen G. Partial information, dominance, and potential optimality in multiattribute utility theory. Operations Research 1986;34(2):296-310.

[21] Iyer N. A family of dominance rules for multiattribute decision making under uncertainty. IEEE Transactions on Systems Man and Cybernetics Part A: Systems and Humans 2003;33:441-50.

[22] Jacquet-Lagrèze E, Siskos Y. Assessing a set of additive utility functions for multicriteria decision making: the UTA method. European Journal of Operational Research 1982;10:151-64.

[23] Keeney R, Raiffa H. Decisions with multiple objectives: preferences and value tradeoffs. New York: John Wiley \& sons; 1976
[24] Lahdelma R, Hokkanen J, Salminen P. SMAA—stochastic multiobjective acceptability analysis. European Journal of Operational Research 1998;106(1):137-43.

[25] Lahdelma R, Miettinen K, Salminen P. Ordinal criteria in stochastic multicriteria acceptability analysis (SMAA). European Journal of Operational Research 2003;147(1):117-27.

[26] Larichev O, Moshkovich H. Zapros: a method and system for ordering multiattribute alternatives on the base of a decision-maker's preferences. Moscow: All-Union Research Institute for Systems Studies, 1991.

[27] Larichev O, Moskovich $H$. An approach to ordinal classification problems. International Transactions in Operational Research 1994;1(3):375-85.

[28] Larichev O, Olson D, Moshkovich $\mathrm{H}$, Mechitov A. Numeric vs. cardinal measurements in multiattribute decision making: how exact is enough?Organizational Behavior and Human Decision Processes 1995;64:9-21.

[29] Lee K, Park K, Kim S. Dominance, potential optimality, imprecise information, and hierarchical structure in multi-criteria analysis. Computers and Operations Research 2002;29:1267-81.

[30] Malakooti B. Ranking and screening multiple criteria alternatives with partial information and use of ordinal and cardinal strength of preferences. IEEE Transactions on Systems Man and Cybernetics: Part A 2000;30(3):787-801.

[31] Marichal J, Meyer P, Roubens M. Sorting multi-attribute alternatives: the TOMASO method. Computers and Operations Research 2005;32(4):861-77.

[32] Mateos A, Rios-Insua S, Jiménez A. Dominance, potential optimality and alternative ranking in imprecise decision making. Journal of Operational Research Society 2007;58(3):326-36.

[33] Moshkovich H, Mechitov A, Olson D. Verbal decision analysis. In: Figueira J, Greco S, Ehrgott M, editors. Multiple criteria decision analysis: state of the art surveys. Boston, Dordrecht, London: Springer Verlag; 2005. p. 609-40.

[34] Paelinck J. Qualiflex: a flexible multiple-criteria decision method. Economic Letters 1978;1:193-7.

[35] Park K. Mathematical programming models for characterizing dominance and potential optimality when multicriteria alternative values and weights are simultaneously incomplete. IEEE Transactions on Systems Man and Cybernetics Part A: Systems and Humans 2004;34:601-14.

[36] Pitz G. DECAID Computer Program, Carbondale, University of Southern Illinois, 1987.

[37] Roubens M. Preference relation on actions and criteria in multicriteria decision making. European Journal of Operational Research 1982;10:51-5.

[38] Roy B, Mousseau V. A theoretical framework for analysing the notion of relative importance of criteria. Journal of Multi-Criteria Decision Analysis 1996;5:145-59.

[39] Sage A, White C. Ariadne: a knowledge-based interactive system for planning and decision support. IEEE Transactions on Systems, Man, and Cybernetics, Part A: Systems and Humans 1984;14:35-47.

[40] Salo A, Hämäläinen R. Preference ratio in multiattribute evaluation PRIME-elicitation and decision procedures under incomplete information. IEEE Transactions on Systems Man and Cybernetics: Part A 2001;31(6): 533-45.

[41] Salo A, Punkka A. Rank inclusion in criteria hierarchies. European Journal of Operational Research 2005;163(2):338-56.

[42] Sarabando P, Dias L. Multi-attribute choice with ordinal information: a comparison of different decision rules. IEEE Transactions on Systems, Man, and Cybernetics: Part A 2009,39(3):545-54.

[43] Solymosi T, Dombi J. A method for determining the weights of criteria: the centralized weights. European Journal of Operational Research 1986;26:35-41.

[44] von Winterfeldt D, Edwards W. Decision analysis and behaviorial research. Cambridge: Cambridge University Press; 1986.

[45] Wakker P. Additive representations of preferences: a new foundation of decision analysis. Dordrecht, Boston, London: Kluwer Academic Publishers; 1989.

[46] Weber M. Decision making with incomplete information. European Journal of Operational Research 1987;28:44-57.

[47] White C, Holloway H. Resolvability for imprecise multiattribute alternative selection. IEEE Transactions on Systems, Man, and Cybernetics, Part A 2008;38(1):162-9. 